\title{
Поправки
}

\section{Эллипсоидальное включение с оболочкой в анизотропной среде с однородным приложенным электрическим полем}

(С) И.В. Лавров, В.Б. Яковлев

В статье, опубликованной в Журнале технической физики (№ 10, 2018 г., С. 1482-1491), были допущены опечатки.

На стр. 1489:

1) левая колонка, строки 16-17 сверху (первая из формул (73)).

Напечатано (пропущена открывающая скобка после $L_{0}^{\prime(1)}$ ):

$$
\begin{aligned}
\boldsymbol{\lambda}_{20}= & {\left[\left(\mathbf{I}+\mathbf{L}_{0}^{\prime(1)} \varepsilon_{1} \mathbf{I}-\boldsymbol{\varepsilon}_{m}\right)\right)\left(\mathbf{I}+3^{-1} \varepsilon_{1}^{-1}\left(1-v^{\prime}\right)\right.} \\
& \left.\left.\times\left(\boldsymbol{\varepsilon}_{2}-\varepsilon_{1} \mathbf{I}\right)\right)+v^{\prime} \mathbf{L}_{0}^{\prime(1)}\left(\boldsymbol{\varepsilon}_{2}-\varepsilon_{1} \mathbf{I}\right)\right]^{-1},
\end{aligned}
$$

Должно быть:

$$
\begin{aligned}
\boldsymbol{\lambda}_{20}= & {\left[( \mathbf { I } + \mathbf { L } _ { 0 } ^ { \prime ( 1 ) } ( \varepsilon _ { 1 } \mathbf { I } - \boldsymbol { \varepsilon } _ { m } ) ) \left(\mathbf{I}+3^{-1} \varepsilon_{1}^{-1}\left(1-v^{\prime}\right)\right.\right.} \\
& \left.\left.\times\left(\boldsymbol{\varepsilon}_{2}-\varepsilon_{1} \mathbf{I}\right)\right)+v^{\prime} \mathbf{L}_{0}^{\prime(1)}\left(\boldsymbol{\varepsilon}_{2}-\varepsilon_{1} \mathbf{I}\right)\right]^{-1}
\end{aligned}
$$

2) левая колонка, строки 13-14 снизу (формула (75)).

Напечатано:

$$
\begin{aligned}
\mathbf{E}_{1}= & \left(-\boldsymbol{\beta}^{(1)}+\varepsilon_{1}^{-1}\left[-3^{-1} \varepsilon_{1}^{-1}+\left(a^{(2)} / r\right)^{3} \mathbf{I}+\left(a^{(2)}\right)^{3}\right.\right. \\
& \left.\left.\times r^{-5}(\mathbf{r} \otimes \mathbf{r})\right] \boldsymbol{\alpha}^{(1)}\right) \mathbf{E}_{0},
\end{aligned}
$$

Должно быть:

$$
\begin{aligned}
\mathbf{E}_{1}= & \left(-\boldsymbol{\beta}^{(1)}+\varepsilon_{1}^{-1}\left[-3^{-1}+\left(a^{(2)} / r\right)^{3} \mathbf{I}+\left(a^{(2)}\right)^{3}\right.\right. \\
& \left.\left.\times r^{-5}(\mathbf{r} \otimes \mathbf{r})\right] \boldsymbol{\alpha}^{(1)}\right) \mathbf{E}_{0},
\end{aligned}
$$

